Pacific Journal of Mathematics

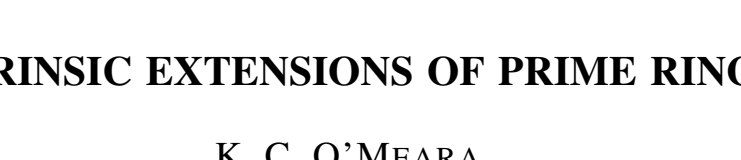




\title{
INTRINSIC EXTENSIONS OF PRIME RINGS
}

\author{
K. C. O'MEARA
}

A ring extension $S$ of a ring $R$ is right intrinsic over $R$, in the sense of Faith and Utumi, if $A \cap R \neq 0$ for each nonzero right ideal $A$ of $S . S$ is a right quotient ring of $R$, in the sense of $\mathbf{R}$. E. Johnson, if $S_{R}$ is an essential extension of $R_{R}$. Let $\mathscr{C}$ be the class of prime rings which have zero right singular ideal and contain uniform right ideals. This paper deals with two questions:

(1) If $S \in \mathscr{C}$ and $S$ is right intrinsic over a prime ring $R$, is $S$ a right quotient ring of $R$ ?

(2) If $R \in \mathscr{C}$ and $S$ is right intrinsic over $R$, is $S$ a right quotient ring of $R$ ?

The main result is that the answer to $(1)$ is "yes" provided $S$ is not an integral domain. As a consequence of this, a partial answer to $(2)$ is "yes" provided $R$ is not an integral domain and $R$ contains a nonzero finite dimensional right annihilator ideal.

An extension of these results to semi-prime rings (which have an atomic lattice of closed right ideals) is also given. These extend results of J. J. Hutchinson (for semi-prime right Goldie rings) and sharpen results of Faith and Utumi for this particular class of rings. A corollary to the main result is that for $R \in \mathscr{C}, R$ not an integral domain, every right quotient ring of $R$ is also a left quotient ring of $R$ if and only if the closed right ideals of $R$ are its right annihilator ideals.

The above corollary can be viewed as a one-sided version of a theorem of Utumi's [10, Theorem 3.3]. The main result, Theorem 2.1, yields another result of interest: A right order in a countably infinite dimensional full linear ring $Q$ must have $Q$ also as a left quotient ring (Theorem 3.4).

To assist the reader to put the results of this paper into perspective, we begin by briefly reviewing what is already known about intrinsic extensions of prime rings.

Let $R$ be a subring of a ring $S$. Following Faith and Utumi [4], we say that $S$ is right (resp. left) intrinsic over $R$ if $A \cap R \neq 0$ for each nonzero right (resp. left) ideal $A$ of $S$. It is clear that if $S$ is a right quotient ring of $R$, then $S$ is right intrinsic over $R$. An interesting question, and one which Faith and Utumi studied in [4], is: When does the converse hold? The main theorem of Faith and Utumi [4, Theorem 3.1], when interpreted for prime rings, can be read as follows: Let $R$ be a prime ring with zero right singular 
ideal but not an integral domain. Suppose that $\left(^{*}\right)$ the maximal right quotient ring of $R$ is left intrinsic over $R$. Then any right intrinsic extension of $R$ is a right quotient ring of $R$. (An intrinsic extension of an integral domain need not be a quotient ring extension; for example, a field is trivially intrinsic over any subfield.) Notice that no finiteness assumptions are required for this theorem. However, the hypothesis $\left({ }^{*}\right)$ is rather restrictive and is not satisfied, for example, even if $R$ is the ring of all $\boldsymbol{\aleph}_{0} \times \boldsymbol{\aleph}_{0}$ column-finite matrices over the rational integers (in which case $R$ contains uniform left ideals as well as uniform right ideals). For a prime right Goldie ring $R, R$ not a right Ore domain, $\left({ }^{*}\right)$ means that $R$ is also left Goldie (see Theorem 3.3). In Theorem 3.7 we replace $\left(^{*}\right)$ by the much weaker hypothesis (for the class of prime rings we consider) that $R$ contain a nonzero finite dimensional right annihilator ideal. A theorem of Hutchinson's [6, Theorem 4.5], when specialized to prime rings, shows that any right intrinsic extension of a prime right Goldie ring $R, R$ not a right Ore domain, is necessarily a right quotient ring of $R$. Theorem 3.7 also covers this result. This summarizes the situation for question (2) of the introductory paragraph. Our principal concern, however, is with question (1). Even in the case of a simple Artinian ring $S, S$ not a division ring, [4] and [6] do not give us an answer to this question. Our answer is given in Theorem 2.1.

Section 1 is devoted to preliminaries. The main result appears in $\S 2$ and its consequences are given in $\S 3$. In $\S 4$ we outline the key steps in extending to semi-prime rings our results for prime rings.

1. Preliminaries. A ring is assumed to be associative but need not have an identity. The unqualified word ideal refers to a twosided ideal.

We shall need a couple of well-known results concerning the closed right ideals and right quotient rings, in the sense of R. E. Johnson, of a ring with zero right singular ideal. These results, together with references of their original source, can be found in Johnson [7]. However, for the reader's convenience, we record them here.

We denote the right singular ideal of a ring $R$ by $Z_{r}(R)$. If $R$ is a subring of a ring $S$, then $S$ is a right (resp. left) quotient ring of $R$ if $S_{R}$ (resp. ${ }_{R} S$ ) is an essential extension of $R_{R}$ (resp. ${ }_{R} R$ ). If $Z_{r}(R)=0$, then $R$ has a unique (up to isomorphism over $R$ ) maximal right quotient ring ( $M R Q$ ring), which is a regular right self-injective ring. A right ideal $I$ of $R$ is called a closed right ideal if $I_{R}$ has no proper essential extensions within $R_{R}$. We denote the lattice of closed right ideals of $R$ by $L_{r}(R)$. 
Proposition 1.1. [7, Corollary 2.6]. Let $R$ be a ring and let $S$ be a right quotient ring of $R$. If $Z_{r}(S)=0$, then $Z_{r}(R)=0$ and $L_{r}(S)$ is isomorphic to $L_{r}(R)$ under the contraction map $A \rightarrow A \cap R$, $A \in L_{r}(S)$.

Let $M_{R}$ be a right module over a ring $R$. For a subset $X$ of $M_{R}$, we denote the annihilator of $X$ in $R$ by $r(X, R)$, that is, $r(X, R)=$ $\{a \in R: X a=0\}$. Analogously, for a left module ${ }_{R} M$ and a subset $X$ of ${ }_{R} M$, we set $l(X, R)=\{a \in R: a X=0\}$.

A module $M_{R}$ is called uniform if it is nonzero and each of its nonzero submodules is essential in $M_{R}$. For a module $M_{R}$ which contains uniform submodules, the uniform dimension of $M_{R}$, denoted by $\operatorname{dim} M_{R}$, is defined to be the cardinal number of any maximal family of independent uniform submodules of $M_{R}$. See Miyashita [8] for an account of this notion.

A ring $Q$ is called a (left) full linear ring if $Q$ is isomorphic to the ring of all linear transformations of some right vector space over a division ring, with transformations written on the left of vectors (equivalently, $Q$ is a prime right self-injective ring with nonzero socle).

Proposition 1.2. [7, Theorem 3.1]. Let $R$ be a prime ring with $Z_{r}(R)=0$ and suppose $R$ contains uniform right ideals. Then the $M R Q$ ring of $R$ is a (left) full linear ring.

The following proposition, due to Utumi [10, Theorem 2.2], shows the nature of condition $\left(^{*}\right)$ in the introduction.

Proposition 1.3. Let $R$ be a ring with $Z_{r}(R)=0$. Then the $M R Q$ ring of $R$ is left intrinsic over $R$ if and only if the closed right ideals of $R$ are its right annihilator ideals.

REMARK. If $R$ has $Z_{r}(R)=0$, then right annihilator ideals of $R$ are closed right ideals.

We conclude our preliminaries by recalling the definition of a right order. A regular element of a ring $R$ is an element $c$ with the property that $l(c, R)=r(c, R)=0$. Let $R$ be a subring of a ring $S$ with identity. Then we say that $R$ is a right order in $S$ if

(i) regular elements of $R$ have (two-sided) inverses in $S$, and

(ii) the elements of $S$ can be expressed in the form $b c^{-1}$, where $b$ and $c$ are in $R$ and $c$ is a unit of $S$.

\section{The main result.}

THEOREM 2.1. Let $S$ be a prime ring with zero right singular 
ideal, but not an integral domain, and suppose $S$ contains uniform right ideals. If $S$ is right intrinsic over a prime ring $R$, then $S$ is a right quotient ring of $R$.

The proof requires several lemmas.

Lemma 2.2. Suppose $Q$ is a simple Artinian ring but not a division ring. Then $Q$ is generated by its idempotents.

Proof. Let $e$ be a primitive idempotent of $Q$. Since $(1-e) Q \neq 0$, $(1-e) Q$ contains a right ideal which is isomorphic to $e Q$. Hence there exist $\beta \in e Q(1-e)$ and $\delta \in(1-e) Q e$ such that $\beta \delta=e$. Let $x \in e Q$. Then

$$
\begin{aligned}
x & =e x e+x(1-e)=\beta(\delta x e)+x(1-e) \\
& =[e+\beta][1-e][e+\delta x e]+[e+x(1-e)][1-e],
\end{aligned}
$$

with each bracketed term an idempotent. Since $Q$ is equal to the sum of its minimal right ideals, it is clear that $Q$, as a ring, is generated by its idempotents.

Lemma 2.3. Let $S$ be a ring with identity, $E$ a set of generators for $S$ (as a ring), and $R$ a subring of $S$ containing units of $S$. Let $U=\{c \in R: c$ a unit in $S\}$ and $T=\{x \in S: x c \in R$ for some $c \in U\}$. If $E \subseteq T$ and $c^{-1} E c \subseteq E$ for all $c \in U$, then $S=T$.

Proof. Let $x \in T$ and $e \in E$. Then there exists $c \in U$ such that $x c \in R$. Choose $c_{1} \in U$ such that $\left(c^{-1} e c\right) c_{1} \in R$. Then $(x e)\left(c c_{1}\right)=$ $(x c)\left(c^{-1} e c\right) c_{1} \in R$. Thus $x e \in T$. Hence finite products of elements in $E$ belong to $T$. If $e_{1}, \cdots, e_{n} \in E$ and $d \in U$, then

$$
\left(e_{1} e_{2} \cdots e_{n}\right) d=d\left(d^{-1} e_{1} d\right) \cdots\left(d^{-1} e_{n} d\right) \in T .
$$

Now let $e_{1}, \cdots, e_{n}$ and $f_{1}, \cdots, f_{m} \in E$. Choose $d \in U$ such that $\left(f_{1} f_{2} \cdots f_{m}\right) d \in R$ and choose $c \in U$ such that $\left(e_{1} \cdots e_{n} d\right) c \in R$. Then $\left(e_{1} e_{2} \cdots e_{n}+f_{1} f_{2} \cdots f_{m}\right) d c \in R$ and thus $e_{1} e_{2} \cdots e_{n}+f_{1} f_{2} \cdots f_{m} \in T$. Since $E$ is a set of generators for $S$, the result is now immediate.

LEMMA 2.4. Let $Q$ be a simple Artinian ring, but not a division ring, and let $R$ be a subring of $Q$. If $R$ is a prime ring and $Q$ is right intrinsic over $R$, then $R$ is a right order in $Q$.

Proof. Let $\operatorname{dim} Q_{Q}=n$. Let $e$ be a primitive idempotent of $Q$ and let $U=e Q \cap R$. Observe that for $a \in U, a \neq 0, Q a$ is a minimal left ideal of $Q$ since $a Q$ is a minimal right ideal of $Q$. Suppose we 
have found $a_{1}, \cdots, a_{m} \in U, m<n$, such that $Q a_{1}+\cdots+Q a_{m}$ is a direct sum. Choose an idempotent $f$ of $Q$ such that $Q a_{1}+\cdots+Q a_{m}=$ $Q f$. Since $Q$ is right intrinsic over $R$ and $R$ is a prime ring, we have $U[(1-f) Q \cap R] \neq 0$. Choose $a_{m+1} \in U$ such that $a_{m+1}(1-f) \neq 0$. Then $Q a_{m+1} \nsubseteq Q f$ and hence $Q a_{m+1} \cap Q f=0$. Thus $Q a_{1}+\cdots+Q a_{m}+$ $Q a_{m+1}$ is a direct sum. Clearly then, there exist $a_{1}, \cdots, a_{n} \in U$ such that $Q a_{1}+\cdots+Q a_{n}=Q$, where + indicates a direct sum. Hence there exist orthogonal primitive idempotents $f_{1}, \cdots, f_{n}$ of $Q$ with $f_{1}+\cdots+f_{n}=1$ and $Q f_{i} \cap R \neq 0$ for $i=1, \cdots, n$.

Now let $g$ be an arbitrary nonzero idempotent of $Q$. Then there exists an integer $k, k \leqq n$, and orthogonal primitive idempotents $g_{1}, \cdots, g_{n}$ of $Q$ such that $g=g_{1}+\cdots+g_{k}$ and $1=g_{1}+\cdots+g_{n}$. Since $g_{i} Q \cap R \neq 0$ and $Q f_{i} \cap R \neq 0$, primeness of $R$ implies $g_{i} Q f_{i} \cap$ $R \neq 0$ for $i=1, \cdots, n$. Choose $c_{i} \in g_{i} Q f_{i} \cap R, c_{i} \neq 0$, for $i=1, \cdots, n$ and let $c=c_{1}+\cdots+c_{n}$. Then $c$ is a unit of $Q$ and $g c=c_{1}+\cdots+$ $c_{k} \in R$. It now follows from Lemmas 2.2 and 2.3 that $R$ is a right order in $Q$.

Lemma 2.5. Suppose $R$ is a prime ring and $Q$ is a full linear ring which is a right quotient ring of $R$. If $f$ is a primitive idempotent of $Q$ such that $Q f \cap R \neq 0$, then $f Q f \cap R$ is a right order in $f Q f$.

Proof. Let $x \in f Q f, x \neq 0$. Choose $r \in R$ such that $f r \in R$ and $0 \neq x r \in R$. Since $R$ is a prime ring, $x r(Q f \cap R) \neq 0$. Hence there exists $y \in f Q f \cap R$ such that $0 \neq x y \in f Q f \cap R$. Since $f Q f$ is a division ring, it is clear that $f Q f \cap R$ is a right order in $f Q f$.

LeMma 2.6. Let $R$ be a subring of a full linear ring $Q$ and suppose $Q$ is right intrinsic over $R$. Let e be a nonzero idempotent of $Q$ and let $K=e Q e \cap R$. If $R$ is a prime ring and $Q e=Q a$ for some $a \in R$, then $K$ is a prime ring and eQe is right intrinsic over $K$.

Proof. Let $x \in e Q e, x \neq 0$. Since $x Q \cap R \neq 0$ and $Q e \cap R \neq 0$, primeness of $R$ implies that $(x Q \cap R)(Q e \cap R) \neq 0$. Hence $x(e Q e) \cap$ $K \neq 0$. Thus $e Q e$ is right intrinsic over $K$. It follows that $Z_{r}(K)=0$. Now suppose $I$ and $J$ are two-sided ideals of $K$ with $I J=0$. Let $L=Q e \cap R$ and $B=e Q \cap R$. Then $(I B)(L J)=0$, which implies $I B=0$ or $L J=0$ because $R$ is a prime ring. $I B=0$ implies $I K=0$, which in turn implies $I=0$ because $Z_{r}(K)=0 . \quad L J=0$ implies $a J=0$, which implies $J=(1-e) J=0$. Thus $K$ is a prime ring and the proof is complete.

Lemma 2.7. Let $Q$ be a full linear ring but not a division ring. 
If $Q$ is right intrinsic over a subring $R$ and $R$ is a prime ring, then $Q$ is a right quotient ring of $R$.

Proof. Let $V$ be a minimal right ideal of $Q$ and let $U=V \cap R$. Choose $u \in U, u \neq 0$. By the argument used in the proof of Lemma 2.4, we can find $v \in U, v \neq 0$, such that $Q u \cap Q v=0$. Choose orthogonal idempotents $f$ and $g$ of $Q$ such that $Q u=Q f$ and $Q v=Q g$. Then $Q f \cap R \neq 0$ and $Q g \cap R \neq 0$. Hence, since $Q$ is right intrinsic over $R$ and $R$ is a prime ring, we have $f Q f \cap R \neq 0$ and $g Q g \cap R \neq 0$. Let $e=f+g$. Then it is clear that there exists $a \in R$ such that $Q a=Q e$. Let $K=e Q e \cap R$. By Lemma 2.6, $K$ is a prime ring and $e Q e$ is right intrinsic over $K$. Hence, since $e Q e$ is a simple Artinian ring but not a division ring, $K$ is a right order in $e Q e$ by Lemma 2.4. By Lemma 2.5, $f K f \cap K$ is a right order in $f(e Q e) f=f Q f$. In particular, $f Q f \cap R$ is a right order in $f Q f$.

Now let $x \in Q f, x \neq 0$. Since $(x Q \cap R)(Q f \cap R) \neq 0$, there exists $b \in f Q f$ such that $0 \neq x b \in Q f \cap R$. $f Q f \cap R$ being a right order in $f Q f$, we can choose $y \in f Q f \cap R$ such that $0 \neq b y \in f Q f \cap R$. Since $Q f$ is a right vector space over $f Q f$, we have $x(b y) \neq 0$ and thus

$$
0 \neq x(b y) \in x(Q f \cap R) \cap(Q f \cap R) .
$$

Hence $Q f$ is a right quotient ring of $Q f \cap R$. Since $Q$ is a prime ring, $Q$ is a right quotient ring of $Q f$ and it follows that $Q$ is therefore a right quotient ring of $R$. This completes the proof of 2.7.

We are now in a position to prove Theorem 2.1.

Proof of Theorem 2.1. Suppose $S$ is right intrinsic over a prime ring $R$. Let $Q$ be the $M R Q$ ring of $S$. Then $Q$ is a left full linear ring by Proposition 1.2, but not a division ring since $S$ is not an integral domain. Clearly $Q$ is also right intrinsic over $R$. Hence $Q$ is a right quotient ring of $R$ by Lemma 2.7, and therefore $S$ is a right quotient ring of $R$. This completes the proof.

\section{Consequences of main result.}

THeOREM 3.1. Let $R$ be a prime ring with $Z_{r}(R)=0$, but not an integral domain, and suppose $R$ contains uniform right ideals. Let $S$ be the MRQ ring of $R$. Then $S$ is a left quotient ring of $R$ if and only if $S$ is left intrinsic over $R$.

Proof. By Proposition 1.2, $S$ is a full linear ring. Hence $S$ has zero left singular ideal and contains uniform left ideals (namely, its minimal left ideals). Clearly $S$ is not an integral domain. Thus, by 
the left-sided version of Theorem 2.1, if $S$ is left intrinsic over $R$ then $S$ is a left quotient ring of $R$. The converse is obvious.

REMARK. For other conditions under which the $M R Q$ ring is also a left quotient ring, see Faith [3, p. 103], Cateforis [1, Theorem 2.3], Cateforis and Sandomierski [2, Theorem 1.1].

We restate Theorem 3.1, using Proposition 1.3, in order to view it as a one-sided version of Utumi's result [10, Theorem 3.3].

THEOREM 3.2. Let $R$ be as in Theorem 3.1. Then every right quotient ring of $R$ is also a left quotient ring of $R$ if and only if the closed right ideals of $R$ are its right annihilator ideals.

A right Goldie ring is a ring which satisfies the maximum condition on closed right ideals and right annihilator ideals. A left Goldie ring is defined analogously. Theorem 3.2 yields the following (probably well-known) criterion of when a prime right Goldie ring is also a left Goldie ring (c.f. [5, Theorems 3.12 and 5.6]).

THEOREM 3.3. Let $R$ be a prime right Goldie ring but not a right Ore domain. Then $R$ is a left Goldie ring if and only if the closed right ideals of $R$ are the right annihilator ideals.

An obvious example of where the one-sided version of Utumi's result [10, Theorem 3.3] fails is obtained by choosing $R$ to be a right Ore domain but not a left Ore domain. In view of the proof of Theorem 2.1, one could possibly attribute the failure in this case to the fact that the $M R Q$ ring of $R$ is not generated by its idempotents. The following is an example of a ring $R$, with $Z_{r}(R)=0$ and $\operatorname{dim} R_{R}=2$, whose $M R Q$ ring is generated by its idempotents but for which the one-sided version of [10, Theorem 3.3] breaks down.

ExAMPLE. Let $K$ be a right Ore domain but not a left Ore domain, and let $D$ be the right quotient division ring of $K$. Let $S$ be the ring of all $2 \times 2$ matrices over $D$ and let $R$ be the subring of $S$ which consists of all matrices of the form

$$
\left(\begin{array}{ll}
k & a \\
0 & b
\end{array}\right) \text {, }
$$

where $k \in K$ and $a, b \in D$. Then $S$ is the $M R Q$ ring of $R$ and $S$ is left intrinsic over $R$, but $S$ is not a left quotient ring of $R$.

Let $Q$ be a left full linear ring. We abbreviate $\operatorname{dim} Q_{Q}$ to $\operatorname{dim} Q$. When $\operatorname{dim} Q$ is finite, our requirement that regular elements of a 
right order $R$ in $Q$ be units of $Q$ is redundant. However, when $\operatorname{dim} Q$ is infinite it implies $Q$ is left intrinsic over $R$. This is shown in [9]. Furthermore, it is shown in [9] that when $\operatorname{dim} Q$ is countable, right orders in $Q$ are prime rings (this being no longer true, in general, when $\operatorname{dim} Q$ is uncountable). Thus by Theorem 3.1 we have:

THEOREM 3.4. Let $R$ be a right order in a left full linear ring $Q$. If $\operatorname{dim} Q$ is countably infinite, then $Q$ is a left quotient ring of $R$. In general, if $\operatorname{dim} Q$ is infinite and $R$ is a prime ring, then $Q$ is a left quotient ring of $R$.

REMARK. If $R$ is a right order in a finite dimensional full linear ring $Q$, then $Q$ is a left quotient ring of $R$ only when $R$ is also a left order in $Q$. This is not necessarily the case for a right order $R$ in an infinite dimensional full linear ring $Q$. For example, let $K_{\infty}$ (resp. $D_{\infty}$ ) be the ring of all $\boldsymbol{\aleph}_{0} \times \boldsymbol{\aleph}_{0}$ column-finite matrices over a right Ore domain $K$ (resp. the right quotient division ring $D$ of $K$ ). Let $Q=D_{\infty}$ and $R=K_{\infty}+\operatorname{socle} Q$. Then $R$ is a right order in $Q$ but not a left order unless each countable collection of nonzero left ideals of $K$ has nonzero intersection.

So far our approach to intrinsic extensions has been to show that certain properties of a prime ring $S$ are inherited by the prime subrings of $S$ over which $S$ is right intrinsic. Our principal applications have called for this approach. The earlier studies by Faith and Utumi [4] and Hutchinson [6] were more concerned with showing that, under suitable conditions on a ring $R$, any ring $S$ which is right intrinsic over $R$ is necessarily a right quotient ring of $R$, thereby showing that certain properties of $R$ are inherited by $S$. We now take a look at intrinsic extensions of prime rings from this point of view.

LEMMA 3.5. Let $S$ be a ring with $Z_{r}(S)=0$ and suppose $S$ is right intrinsic over a subring $R$. Then:

(i) For $x, y \in S, x y S \neq 0$ implies $x(y S \cap R) \neq 0$.

(ii) If $R$ contains uniform right ideals, then so does $S$.

Proof. (i ) Suppose $x y S \neq 0$. Let $K=\{a \in S: x a=0\}$. If $y S \cap K$ is essential in $y S$, as a right $S$-module, then $Z_{r}(S)=0$ and $x(y S \cap K)=0$ imply $x y S=0$, a contradiction. Thus $y S \cap K$ is not essential in $y S$ and therefore there exists a nonzero right ideal $A$ of $S$ such that $A \subseteq y S$ and $A \cap K=0$. Since $S$ is right intrinsic over $R$ we have $A \cap R \neq 0$. Hence $x(A \cap R) \neq 0$, since $A \cap K=0$. Thus $x(y S \cap R) \neq 0$.

(ii) Suppose $U$ is a uniform right ideal of $R$. Choose $x \in U$, $x \neq 0$. Then $x S$ is a uniform right ideal of $S$. For if not, then 
$x y S \cap x z S=0$ for some $y, z \in S$ with $x y \neq 0$ and $x z \neq 0$. By (i), $x(y S \cap R) \neq 0$ and $x(z S \cap R) \neq 0$. But now

$$
[x(y S \cap R)] \cap[x(z S \cap R)]=0,
$$

which contradicts the fact that $U$ is a uniform right ideal of $R$. This completes the proof of the lemma.

LEMMA 3.6. Let $R$ be a prime ring with $Z_{r}(R)=0$ and suppose $R$ contains a uniform right ideal and a nonzero right annihilator ideal $B$ with $\operatorname{dim} B_{R}<\infty$. Then there exists an element $y \in R$ such that $r(y, R) \cap B=0$.

REMARK. $R$ need not contain an element $y$ with $r(y, R)=0$.

Proof. Let $Q$ be the $M R Q$ ring of $R$. Then $Q$ is a full linear ring by Proposition 1.2. Choose an idempotent $e$ of $Q$ such that $B=$ $e Q \cap R$. By Proposition 1.1, we have $\operatorname{dim} B_{R}=\operatorname{dim}(e Q)_{Q}$ and hence $\operatorname{dim}(e Q)_{Q}<\infty$. Let $\operatorname{dim}(e Q)_{Q}=n$. By an argument similar to that used in the proof of Lemma 2.4, we can find nonzero elements $a_{1}, \cdots, a_{n}$ of $R$ such that each $Q a_{i}$ is a minimal left ideal of $Q$ and

$$
Q=Q(1-e)+Q a_{1}+\cdots+Q a_{n},
$$

where + indicates a direct sum. Choose orthogonal primitive idempotents $g_{1}, \cdots, g_{n}$ of $Q$ such that $Q g_{i}=Q \alpha_{i}$ for $i=1, \cdots, n$. Since $g_{i} Q \cap R \neq 0$, we have $\left(g_{i} Q \cap R\right) a_{i} \neq 0$ by primeness of $R$. Thus $g_{i} Q g_{i} \cap$ $R \neq 0$ for $i=1, \cdots, n$. Choose $y_{i} \in g_{i} Q g_{i} \cap R, y_{i} \neq 0$, for $i=1, \cdots, n$ and let $y=y_{1}+\cdots+y_{n}$. Then $Q=Q(1-e)+Q y$ and hence $r(y, Q) \cap$ $e Q=0$. In particular, $r(y, R) \cap B=0$.

It is not known to the author whether a prime ring which has zero right singular ideal and contains uniform right ideals must contain a nonzero finite dimensional right annihilator ideal. (Conjecture: yes.) The answer is "yes" if the ring also contains uniform left ideals because then every finite dimensional closed right ideal is a right annihilator ideal (see Utumi [11, Proposition 7.3]).

THEOREM 3.7. Let $R$ be a prime ring with $Z_{r}(R)=0$, but not an integral domain, and suppose $R$ contains a uniform right ideal and a nonzero right annihilator ideal $B$ with $\operatorname{dim} B_{R}<\infty$. Then any ring $S$ which is right intrinsic over $R$ is necessarily a right quotient ring of $R$ (and therefore lies between $R$ and the MRQ ring of $R$, which is a full linear ring).

Proof. It is clear that $S$ is a prime ring but not an integral domain. If we can show that $Z_{r}(S)=0$ then it will follow from 
Lemma 3.5 that $S$ contains uniform right ideals and hence, by Theorem 2.1, that $S$ is a right quotient ring of $R$. Let us suppose that $Z_{r}(S) \neq 0$. Let $I=Z_{r}(S) \cap R$. Then $I$ is a nonzero ideal of $R$. As such, $I$ is also a prime ring with $Z_{r}(I)=0$ and $I$ contains uniform right ideals. Let $B$ be a nonzero finite dimensional right annihilator ideal of $R$. Then $I \cap B$ is a nonzero finite dimensional right annihilator ideal of $I$. Applying Lemma 3.6 to $I$, we can find an element $y \in I$ and a left ideal $L$ of $I$ such that $r(L, I) \neq 0$ and $r(y, I) \cap$ $r(L, I)=0$. Let $A=r(L, S)$. Then $A$ is a nonzero right ideal of $S$ and since $y \in Z_{r}(S)$ we have $A \cap r(y, S) \neq 0$. Moreover, since $S$ is right intrinsic over $R$, and hence over $I$, we have $A \cap r(y, S) \cap I \neq 0$, that is, $r(y, I) \cap r(L, I) \neq 0$. With this contradiction, we deduce that $Z_{r}(S)=0$. We are finished.

Hutchinson [6, Theorem 4.5] characterized intrinsic extensions of semi-prime right Goldie rings. As a corollary to Theorem 3.7 we obtain Hutchinson's result when specialized to prime right Goldie rings.

CoROLlaRY 3.8. Let $R$ be a prime right Goldie ring, but not a right Ore domain. Then any ring which is right intrinsic over $R$ is necessarily a right quotient ring of $R$ (and therefore lies between $R$ and the classical right quotient ring of $R$, which is a simple Artinian ring).

Remark. Faith and Utumi [4, Theorem 3.2] proved this under the assumption that $R$ is also a left Goldie ring.

4. Intrinsic extensions of semi-prime rings. Let $R$ be a ring with $Z_{r}(R)=0$. The lattice $L_{r}(R)$ of closed right ideals of $R$ is said to be atomic if each nonzero element of $L_{r}(R)$ contains a minimal nonzero element (an atom) of $L_{r}(R)$. If $R$ is a prime ring containing a uniform right ideal, then $L_{r}(R)$ is atomic. Our results on intrinsic extensions for such rings can be extended to semi-prime rings $S$ which have $Z_{r}(S)=0$ and for which $L_{r}(S)$ is atomic by using the fact that the $M R Q$ ring of $S$ is a (complete) direct product of left full linear rings (see Johnson [7, Theorem 3.1]). Thus, if $T$ is the $M R Q$ ring of $S$, there exists a set $\left\{e_{i}\right\}_{i \in I}$ of central orthogonal idempotents of $T$ such that each $e_{\imath} T$ is a left full linear ring and $T$ is isomorphic to $\prod_{i \in I} e_{i} T$ under the map $x \rightarrow\left(e_{i} x\right)$. Now suppose $S$ is right intrinsic over a semi-prime ring $R$. For each $i \in I$, let $T_{i}=e_{i} T$ and $R_{\imath}=T_{i} \cap R$. We make the following

Claim. (1) $T_{i}$ is right intrinsic over $R_{\imath}$ for each $i \in I$.

(2) Each $R_{i}$ is a prime ring. 
(3) If $T_{i}$ is a right quotient ring of $R_{i}$ for each $i \in I$, then $T$ (and hence $S$ ) is a right quotient ring of $R$.

(1) and (3) are easily verified. We shall now verify (2). It is clear that each $R_{i}$ is at least semi-prime because it is a nonzero ideal of a semi-prime ring. That each $R_{i}$ is in fact a prime ring is the content of the following lemma.

Lemma 4.1. Suppose $Q$ is a full linear ring which is right intrinsic over a semi-prime ring $A$. Then $A$ is a prime ring.

Proof. Let us suppose $A$ is not a prime ring. Then there exist nonzero ideals $I$ and $J$ of $A$ with $I J=0$. We can suppose $J=r(I, A)$ and $I=l(J, A)$. Since $A$ is semi-prime, we have $I=r(J, A)$ and $I \cap J=0$. Let $I_{1}=r(J, Q)$ and $J_{1}=r(I, Q)$. Then $I=I_{1} \cap A$ and $J=J_{1} \cap A$. Hence, since $Q$ is right intrinsic over $A$ and $I \cap J=0$, we have $I_{1} \cap J_{1}=0$. Since $Q$ is a full linear ring, we can choose a nonzero right ideal $K_{1}$ of $Q$ such that $I_{1} \cap K_{1}=0$ and $J_{1} \cap K_{1}=0$. Let $K=K_{1} \cap A$. Then $K \neq 0$ since $Q$ is right intrinsic over $A$. But $J \cap K=0$ implies $K J=0$ and therefore $K \subseteq I \cap K=0$, that is, $K=0$. We have the desired contradiction. Hence $A$ is a prime ring.

Providing none of the $T_{i}$ are division rings, we can apply Theorem 2.1 to obtain that $T_{i}$ is a right quotient ring of $R_{i}$ for each $i \in I$. It then follows from (3) that $S$ is a right quotient ring of $R$. To eliminate the possibility of a division ring appearing among the $T_{i}$, we shall require that $S$ contains no ideals which, as rings, are integral domains. Summarizing, we have the following extension of Theorem 2.1.

THEOREM 4.2. Let $S$ be a semi-prime ring with $Z_{r}(S)=0$, but containing no ideals which are integral domains, and suppose $L_{r}(S)$ is atomic. Then $S$ is a right quotient ring of each semi-prime ring over which it is right intrinsic.

As a corollary to Theorem 4.2 we have the following partial extension of Theorem 3.7.

COROLlary 4.3. Let $R$ be a semi-prime ring with $Z_{r}(R)=0$, but containing no ideals which are integral domains, and suppose $L_{r}(R)$ is atomic. If $S$ is a ring which is right intrinsic over $R$ and $Z_{r}(S)=0$, then $S$ is a right quotient ring of $R$.

Proof. All we need show is that $L_{r}(S)$ is atomic, equivalently, that each nonzero right ideal of $S$ contains a uniform right ideal. Let $I$ be a nonzero right ideal of $S$. Then $I \cap R$ is a nonzero right ideal of $R$ and hence contains a uniform right ideal $U$ of $R$. The 
proof of Lemma 3.5 shows that for $x \in U, x \neq 0, x S$ is a uniform right ideal of $S$. Since $x S \subseteq I$, this establishes the corollary.

REMARKS. (1) If $R$ is a semi-prime right Goldie ring and $S$ is a ring which is right intrinsic over $R$, then $Z_{r}(S)=0$ (c.f. [6, Theorem 4.6]). To see this, we argue as follows. $R$ contains nonzero ideals $R_{1}, \cdots, R_{n}$ such that each $R_{i}$ is a prime right Goldie ring and the sum $R_{1}+\cdots+R_{n}$ is direct and an essential right ideal of $R$. (By Corollary 3.8 we can assume $n \geqq 2$.) Hence $S$ is also right intrinsic over $R_{1}+\cdots+R_{n}$. Now suppose $Z_{r}(S) \neq 0$. Let $I=Z_{r}(S) \cap R$. Then $I$ is a nonzero ideal of $R$ and $I R_{i} \neq 0$ for some $i$. Without loss of generality we can suppose $I R_{1} \neq 0$. Let $J=I R_{1}$. Then $J$ is a nonzero ideal of $R_{1}$ and therefore there exists $y \in J$ such that $r\left(y, R_{1}\right)=0$. Since $y \in Z_{r}(S)$ we must have $r(y, S) \cap r\left(R_{2}+\cdots+R_{n}, S\right) \neq 0$ and therefore

$$
r(y, S) \cap r\left(R_{2}+\cdots+R_{n}, S\right) \cap\left(R_{1}+\cdots+R_{n}\right) \neq 0,
$$

that is, $r\left(y, R_{1}\right) \neq 0$. We have reached a contradiction. Hence $Z_{r}(S)=0$.

(2) Corollary 4.3 can be obtained in the special case of a semiprime right Goldie ring $R$ from Theorems 3.4 and 4.5 of Hutchinson [6].

(3) For a ring $R$ which has $Z_{r}(R)=0$ and $L_{r}(R)$ atomic, the condition that $R$ contain no ideals which are integral domains is equivalent to the condition that the $M R Q$ ring of $R$ contain no nonzero strongly regular ideals. Thus Corollary 4.3 represents a considerable strengthening of [4, Theorem 3.1] in the case where $R$ is semi-prime. Simple examples, such as the ring $R$ of all $2 \times 2$ matrices of the form

$$
\left(\begin{array}{ll}
a & b \\
0 & c
\end{array}\right)
$$

where $a$ and $b$ belong to a field $D$ and $c$ belongs to a proper subfield $F$ of $D$, show that without semi-primeness of $R$ Corollary 4.3 is, in general, false. (In the example cited, $\operatorname{dim} R_{R}=\operatorname{dim} D_{F}+1$.)

(4) It is clear that theorems corresponding to Theorems 3.1, 3.2 , and 3.3 can be formulated for semi-prime rings. The details may safely be left to the reader.

\section{REFERENCES}

1. V. C. Cateforis, Two-sided semisimple maximal quotient rings, Trans. Amer. Math. Soc., 149 (1970), 339-349.

2. V. C. Cateforis and F. L. Sandomierski, On modules of singular submodule zero, Canad. J. Math., 23 (1971), 345-354. 
3. C. Faith, Lectures on Injective Modules and Quotient Rings, Lecture Notes in Math., no. 49, Springer-Verlag, New York, 1967.

4. C. Faith and Y. Utumi, Intrinsic extensions of rings, Pacific J. Math., 14 (1964), 505-512.

5. A. W. Goldie, Semi-prime rings with maximum condition, Proc. London Math. Soc., 10 (1960), 201-220.

6. J. J. Hutchinson, Intrinsic extensions of rings, Pacific J. Math., 30 (1969), 669-677.

7. R. E. Johnson, Quotient rings of rings with zero singular ideal, Pacific J. Math., 11 (1961), 1385-1392.

8. Y. Miyashita, On quasi-injective modules, J. Fac. Sci. Hokkaido Univ. Ser. I, 18 (1965), 158-187.

9. K. C. O'Meara, Primeness of right orders in full linear rings, to appear in J. Algebra.

10. Y. Utumi, On rings of which any one-sided quotient rings are two-sided, Proc. Amer. Math. Soc., 14 (1963), 141-147.

11. - On prime J-rings with uniform one-sided ideals, Amer. J. Math., 85 (1963), 583-596.

Received December 18, 1972 and in revised form April 13, 1973. This paper is based on a portion of the author's Ph.D. dissertation written under the supervision of Dr. Robert L. Kruse at the University of Canterbury. The author wishes to express his gratitude to Dr. Kruse for his valuable help and encouragement.

University of Canterbury, Christchurch, New Zealand 



\section{PACIFIC JOURNAL OF MATHEMATICS}

\section{EDITORS}

RICHARD ARENS (Managing Editor)

University of California

Los Angeles, California 90024

R. A. BeAumont

University of Washington

Seattle, Washington 98105
J. DugundJI*

Department of Mathematics

University of Southern California

Los Angeles, California 90007

D. Gilbarg and J. Milgram

Stanford University

Stanford, California 94305

\section{ASSOCIATE EDITORS}

E. F. BECKENBACH

B. H. NEUMANN

F. WOLF

K. YoSHIDA

\section{SUPPORTING INSTITUTIONS}

UNIVERSITY OF BRITISH COLUMBIA
CALIFORNIA INSTITUTE OF TECHNOLOGY
UNIVERSITY OF CALIFORNIA
MONTANA STATE UNIVERSITY
UNIVERSITY OF NEVADA
NEW MEXICO STATE UNIVERSITY
OREGON STATE UNIVERSITY
UNIVERSITY OF OREGON
OSAKA UNIVERSITY

UNIVERSITY OF BRITISH COLUMBIA CALIFORNIA INSTITUTE OF TECHNOLOGY UNIVERSITY OF CALIFORNIA MONTANA STATE UNIVERSITY NEW MEXICO STATE UNIVERSITY UNIVERSITY OF OREGON OSAKA UNIVERSITY
UNIVERSITY OF SOUTHERN CALIFORNIA STANFORD UNIVERSITY UNIVERSITY OF TOKYO UNIVERSITY OF UTAH WASHINGTON STATE UNIVERSITY UNIVERSITY OF WASHINGTON AMERICAN MATHEMATICAL SOCIETY NAVAL WEAPONS CENTER

* C. R. DePrima California Institute of Technology, Pasadena, CA 91109, will replace J. Dugundji until August 1974. 


\section{Pacific Journal of Mathematics}

\section{Vol. 51, No. $1 \quad$ November, 1974}

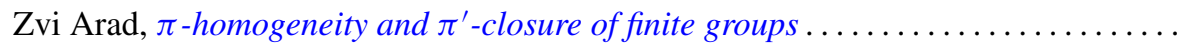

Ivan Baggs, A connected Hausdorff space which is not contained in a maximal

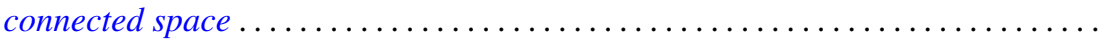

Eric Bedford, The Dirichlet problem for some overdetermined systems on the unit ball in $C^{n}$

R. H. Bing, Woodrow Wilson Bledsoe and R. Daniel Mauldin, Sets generated by

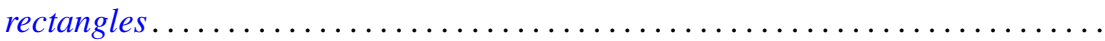

Carlo Cecchini and Alessandro Figà-Talamanca, Projections of uniqueness for

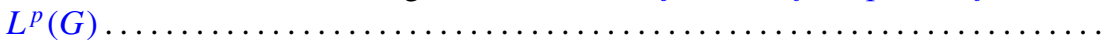

Gokulananda Das and Ram N. Mohapatra, The non absolute Nörlund summability of Fourier series .

Frank Rimi DeMeyer, On separable polynomials over a commutative ring ........ Richard Detmer, Sets which are tame in arcs in $E^{3} \ldots \ldots \ldots \ldots \ldots \ldots \ldots \ldots$

William Erb Dietrich, Ideals in convolution algebras on Abelian groups ..........

Bryce L. Elkins, A Galois theory for linear topological rings .................

William Alan Feldman, A characterization of the topology of compact convergence on $C(X)$.

Hillel Halkin Gershenson, A problem in compact Lie groups and framed cobordism

Samuel R. Gordon, Associators in simple algebras.

Marvin J. Greenberg, Strictly local solutions of Diophantine equations

Jon Craig Helton, Product integrals and inverses in normed rings . . . . . . . . . . . .

Domingo Antonio Herrero, Inner functions under uniform topology . . .

Jerry Alan Johnson, Lipschitz spaces .

Marvin Stanford Keener, Oscillatory solutions and multi-point boundary value

functions for certain nth-order linear ordinary differential equations.

John Cronan Kieffer, A simple proof of the Moy-Perez generalization of the

Shannon-McMillan theorem .......................

Joong Ho Kim, Power invariant rings

Gangaram S. Ladde and V. Lakshmikantham, On flow-invariant sets .

Roger T. Lewis, Oscillation and nonoscillation criteria for some self-adjoint even

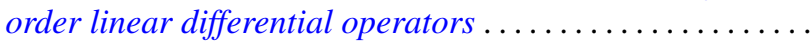

Jürg Thomas Marti, On the existence of support points of solid convex sets ..

John Rowlay Martin, Determining knot types from diagrams of knots . .

James Jerome Metzger, Local ideals in a topological algebra of entire functions

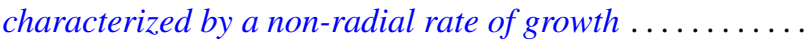

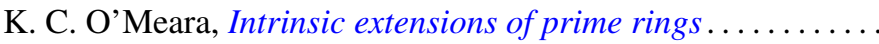

Stanley Poreda, A note on the continuity of best polynomial approximations ..

Robert John Sacker, Asymptotic approach to periodic orbits and local prolongations

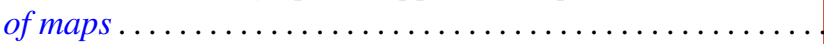

Eric Peter Smith, The Garabedian function of an arbitrary compact set . .

Arne Stray, Pointwise bounded approximation by functions satisfying a side condition

John St. Clair Werth, Jr., Maximal pure subgroups of torsion complete abelian

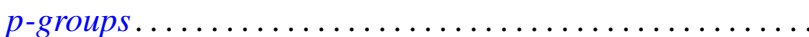

